\title{
Assessment of Arterial Stiffness Index in Hypertensive Patients in Relation to Their Treatment Status Attending a Tertiary Care Center in South India
}

\author{
Shourya Kola1 (D), Melvin George², Suresh Kumar Srinivasamurthy3, Sandhiya Selvarajan³, \\ Kadhiravan Tamilarasu4, Rathinam Palamalai Swaminathan ${ }^{4}$, Adithan Chandrasekaran ${ }^{3}$ \\ ${ }^{1}$ Jawaharlal Institute of Postgraduate Medical Education \& Research, Puducherry, India \\ ${ }^{2}$ Cardiac Clinical Trials and Research, Department of Cardiology, SRM Medical College, Hospital and Research \\ Centre, Chennai, India \\ ${ }^{3}$ Department of Clinical Pharmacology, Jawaharlal Institute of Postgraduate Medical Education \& Research, \\ Puducherry, India \\ ${ }^{4}$ Department of Medicine, Jawaharlal Institute of Postgraduate Medical Education \& Research, Puducherry, India \\ Email: ksvtej@gmail.com, melvingeorge2003@gmail.com, sureshjalapalli@gmail.com, \\ sandhiyaselvarajan@gmail.com, kadhiravan@gmail.com, swaminathan.rp@jipmer.edu.in, adithan50@gmail.com
}

Received 21 January 2014; revised 19 March 2014; accepted 9 April 2014

Copyright @ 2014 by authors and Scientific Research Publishing Inc.

This work is licensed under the Creative Commons Attribution International License (CC BY). http://creativecommons.org/licenses/by/4.0/

(c) (i)

\section{Abstract}

Objectives: To assess the arterial stiffness index (ASI) and pulse wave velocity (PWV) in patients with hypertension and to compare with age matched healthy controls; to assess and compare the ASI and PWV in relation to the treatment status. Methods: The study was observational-cross sectional. Group one included chronic hypertensive patients on regular treatment for more than 2 months; group two included newly diagnosed hypertensive patients and group three had age matched healthy controls with normal blood pressure. The hypertensives subjects with other comormid conditions such as renal disease, diabetes were excluded from the study. The study was approved by the Institute Ethics Committee. The subjects were interviewed and explained the purpose of the study. All subjects gave written informed consent. The noninvasive periscope device was used to measure PWV, ASI and pulse pressure. Results: PWV, ASI and pulse pressure were statistically higher in hypertensive patients when compared to controls. Further, carotid-femoral PWV was correlated with mean arterial pressure in hypertensive subjects and was found to be statistically significant. Conclusion: PWV, ASI and pulse pressure are significantly higher in chronic and newly diagnosed non-diabetic hypertensives as compared to controls irrespective of their treatment status.

How to cite this paper: Kola, S., George, M., Srinivasamurthy, S.K., Selvarajan, S., Tamilarasu, K., Swaminathan, R.P. and Chandrasekaran, A. (2014) Assessment of Arterial Stiffness Index in Hypertensive Patients in Relation to Their Treatment Status Attending a Tertiary Care Center in South India. Pharmacology \& Pharmacy, 5, 413-418. 


\section{Keywords}

\section{Pulse Wave Velocity; Arterial Stiffness Index; Hypertension; Antihypertensives}

\section{Introduction}

Cardiovascular diseases are emerging as one of the leading causes of morbidity and mortality in the Indian population. By 2020, 2.6 million deaths are predicted to be due to coronary heart disease among Indians [1]. The major risk factors contributing to cardiovascular diseases include hypertension, diabetes mellitus, smoking, hypercholesterolemia and family history of coronary artery disease. Among the various risk factors, hypertension contributes to nearly 7.1 million premature deaths, two-thirds of all strokes and half of myocardial infarction every year [2]. The worldwide prevalence of hypertension in 2000 was $26.4 \%$ including approximately a quarter of the adult population and it is estimated to rise to $29.2 \%$ by 2025 [3]. In addition to the occurrence of end organ damage, the uncontrolled rise in arterial pressure has been found to be associated with progressive increase in the risk of cardiovascular events [4]. Arterial stiffness, a major contributor of atherosclerosis, has been shown to have positive relationship with systolic hypertension, coronary artery disease, stroke and heart failure. Further, aortic stiffness has been shown to be an independent predictor of all-cause and cardiovascular mortality in hypertensive patients. In the present scenario, arterial stiffness and the ensuing hemodynamic changes are recognized as imperative predictors of adverse cardiovascular events. Hence measurement of arterial stiffness seems to be a promising tool to assess cardiovascular risk in hypertensive patients [5] [6].

The various measures of arterial stiffness used as predictors of cardiovascular morbidity and mortality include pulse wave velocity (PWV), augmentation index (AI), arterial stiffness index (ASI), pulse pressure (PP) [7]. Among these parameters, pulse wave velocity (PWV), pulse pressure (PP) and arterial stiffness index (ASI) have established their predictive accuracy in cardiovascular morbidity and mortality [8]-[11]. Pulse wave velocity and arterial stiffness index as assessed by an instrument termed "periscope" has been shown to be a simple measure of arterial stiffness in population studies [12]. In addition, PWV, considered as a gold standard to measure the arterial stiffness, has been used to establish the vascular effects of antihypertensive therapy [6] [13]. Evidence shows that angiotensin converting enzyme (ACE) inhibitors, angiotensin receptor blockers (ARBs), aldosterone receptor antagonists and calcium channel blockers have favorable effect on improving arterial stiffness compared to beta blockers [5] [14]. However, at present there are no guidelines regarding the use of anti-hypertensive drugs to target arterial stiffness. In addition, the benefit of monitoring these vascular parameters in hypertensive patients remains unanswered. As there is a need to collect data on the vascular parameters of stiffness in hypertensives compared to the healthy subjects, the study was done to measure PWV, ASI and pulse pressure in hypertensive patients. The study also aimed to determine the effect of antihypertensive drugs on these vascular parameters.

\section{Objectives}

1) To assess the arterial stiffness index and pulse wave velocity in patients with hypertension and to compare with age matched healthy controls.

2) To assess and compare the arterial stiffness index and pulse wave velocity in relation to the treatment status of hypertensive patients.

\section{Materials \& Methods}

The study was an observational-cross sectional study of three groups with group one of chronic hypertensive patients on regular treatment for more than 2 months; group two included newly diagnosed hypertensive patients and group three comprised of age matched healthy controls with normal blood pressure.

Eligible patients and healthy controls were recruited from the medicine outpatient department (OPD) after obtaining written informed consent. The subjects were interviewed and explained about the purpose of the study. All patients were aged $40-60$ years and belonged to either gender. Chronic hypertensives on drug therapy for at least 2 months with systolic blood pressure (SBP) less than $180 \mathrm{mmHg}$ or diastolic blood pressure (DBP) less 
than $110 \mathrm{mmHg}$ were included in group one. Newly diagnosed hypertensives with SBP in the range of $140-180$ $\mathrm{mmHg}$ or diastolic blood pressure in range of $90-110 \mathrm{mmHg}$ were included in group two. Subjects with blood pressure in the normal range were included in group three. Cases and controls with co-morbid medical illness such as diabetes, stroke and presence of advanced renal disease (serum creatinine $>2.5 \mathrm{mg} / \mathrm{dl}$ ) were excluded from the study.

After recording of anthropometric data and brief history, subjects were asked to rest in supine position on a couch for five minutes. Care was taken to ensure that subjects did not smoke, or consume food or caffeinated beverages two hours prior to the study. The non-invasive periscope device was used to measure PWV, ASI and pulse pressure. The recordings were taken by applying pneumatic cuffs around the four limbs and electrocardiograph (ECG) electrodes were placed on the chest. A minimum of three readings of PWV, ASI and pulse pressure were taken and the means were calculated. The study was approved by the Institute Ethics Committee, JIPMER, Puducherry.

\section{Statistical Analysis}

Sample size $(\mathrm{N}=120)$ was calculated to be forty in each group based on convenience sampling. All categorical data (gender, smoking status etc.) were presented as percentages. The data related to vascular parameters of the study (PWV, ASI and pulse pressure) were presented as mean \pm standard deviation (SD). The categorical data between the groups were compared using chi square test. The PWV, ASI and pulse pressure were compared by using one way analysis of variance (ANOVA) with post hoc analysis or Kruskal Wallis test whichever was appropriate. Statistical analysis was carried out at $5 \%$ level of significance and $\mathrm{P}$ value $<0.05$ was considered significant. Correlation between the parameters of stiffness with mean arterial pressure among hypertensives was done using Spearman rank correlation.

\section{Results}

Demographic characteristics including age, gender, body mass index, smoking status and alcoholic status were not statistically different among three groups. However, mean blood pressure, systolic blood pressure, diastolic blood pressure were significantly higher in hypertensives as compared to control group (Table 1). In the present study, PWV, ASI and pulse pressure were found to be statistically higher in hypertensive patients when compared to control group (Table 2). Further, carotid-femoral PWV correlated with mean arterial pressure in hypertensive subjects was found to be statistically significant $(r=0.40)$. The chronic hypertensive patients were grouped based on the drug therapy received. Among the 40 patients in group one, thirteen were on monotherapy which included amlodipine and the remaining were on polytherapy that included ACE inhibitors, beta blockers and statins. Comparison of vascular parameters between the patients receiving amlodipine monotherapy and polytherapy did not show statistically significant difference (Table 3).

\section{Discussion}

In our study, the vascular parameters namely PWV, ASI and pulse pressure were significantly higher in hypertensive subjects as compared to the controls. These findings were in accordance with a previous study done to assess the validity and reproducibility of arterial PWV using oscillometric technique [12]. Emerging data suggests that carotid femoral pulse wave velocity is a gold standard for noninvasive evaluation of aortic stiffness [6]. Hence in the present study, carotid femoral PWV was correlated with mean arterial pressure in hypertensives. The correlation obtained between carotid femoral pulse wave velocity and mean arterial pressure was also in accordance with the previous study which showed higher PWV with higher mean arterial pressure [12]. The higher blood pressure observed in hypertensives (Table 1) may be due to lack of adherence to the antihypertensive drugs, which was not ensured objectively as this was a cross sectional study with no follow-ups.

Chronic hypertensive subjects were on combination of drug therapy and only thirteen patients were on monotherapy with amlodipine. Hence comparison was done between amlodipine monotherapy group and polytherapy group with reference to vascular parameters obtained. There was no statistical significance between these groups inferring that arterial stiffness was equally high in both these sub-groups.

Aging is well recognized as a risk factor for arterial stiffening [15]. Increased arterial stiffening with aging is also associated with raised blood pressure and pulse pressure, thus showing the inter-relation among these parameters. In our study, the age was not significantly different among the three groups. Nevertheless, PWV, ASI 
Table 1. Demographic and other characteristics of study subjects.

\begin{tabular}{|c|c|c|c|}
\hline Characteristic & Chronic hypertensives $(N=40)$ & New hypertensives $(N=40)$ & Controls $(\mathrm{N}=40)$ \\
\hline Age (years) & $51.5 \pm 6.46$ & $50.6 \pm 6.32$ & $49.7 \pm 6.22$ \\
\hline Gender: Male & $22(55 \%)$ & $27(68 \%)$ & $26(65 \%)$ \\
\hline Female & $18(45 \%)$ & $13(32 \%)$ & $14(35 \%)$ \\
\hline Body mass index $\left(\mathrm{kg} / \mathrm{m}^{2}\right)$ & $25.1 \pm 4.74$ & $25 \pm 5.03$ & $23.3 \pm 5.29$ \\
\hline Smokers & $7(17.5 \%)$ & $10(25 \%)$ & $4(10 \%)$ \\
\hline Alcoholics & $8(20 \%)$ & $14(35 \%)$ & $7(17.5 \%)$ \\
\hline Mean blood pressure $(\mathrm{mmHg})$ & $102.9 \pm 10.83$ & $107.47 \pm 11.83$ & $88.0 \pm 13.87^{*}$ \\
\hline Mean duration (years) & $3.18 \pm 4.43$ & - & - \\
\hline $\mathrm{SBP}(\mathrm{mmHg})$ & $142.32 \pm 18.61$ & $148.78 \pm 17.95$ & $120.95 \pm 12.58^{*}$ \\
\hline DBP (mmHg) & $83.32 \pm 9.16$ & $90.2 \pm 7.1$ & $74 \pm 8.53^{*}$ \\
\hline
\end{tabular}

Data are presented as mean \pm SD and percentages given in parenthesis for categorical data; $\mathrm{N}=$ sample size in the group; ANOVA was used for comparison of data among the groups; Chi square test used for comparison of gender, smokers and alcoholics among the groups; ${ }^{*} \mathrm{P}<0.05$.

\section{Table 2. PWV, ASI and pulse pressure among hypertensive and control groups.}

\begin{tabular}{cccc}
\hline Parameter & Chronic hypertensives $(\mathrm{N}=40)$ & Hypertensives $(\mathrm{N}=40)$ & Controls $^{*}(\mathrm{~N}=40)$ \\
\hline Carotid femoral PWV $(\mathrm{cm} / \mathrm{s})$ & $1017.48 \pm 416.67$ & $1004.69 \pm 519.26$ & $704.25 \pm 328.45$ \\
Left brachial PWV $(\mathrm{cm} / \mathrm{s})$ & $1067.63 \pm 1521.2$ & $1345.78 \pm 834.53$ & $967.04 \pm 630.14$ \\
Right brachial PWV $(\mathrm{cm} / \mathrm{s})$ & $1508.73 \pm 575.22$ & $1491.67 \pm 625.52$ & $1244.03 \pm 530.07$ \\
Right brachial ASI (mmHg) & $23.33 \pm 12.66$ & $27.74 \pm 11.7$ & $20.00 \pm 7.37$ \\
Left brachial ASI (mmHg) & $27.61 \pm 7.61$ & $29.01 \pm 11.68$ & $23.83 \pm 6.08$ \\
Right ankle ASI (mmHg) & $36.32 \pm 10.38$ & $35.76 \pm 9.57$ & $30.89 \pm 11.96$ \\
Left ankle ASI (mmHg) & $39.31 \pm 8.53$ & $36.99 \pm 8.65$ & $31.28 \pm 6.81$ \\
Pulse pressure (mmHg) & $59.0 \pm 15.58$ & $60.95 \pm 13.52$ & $47.58 \pm 8.45$ \\
\hline
\end{tabular}

PWV: pulse wave velocity; ASI: arterial stiffness index; data are presented as mean $\pm \mathrm{SD}$; Kruskal Wallis test was used for comparison of the data; ${ }^{*} \mathrm{P}$ value $<0.05$.

Table 3. Comparison of vascular parameters between the patients receiving amlodipine as monotherapy and along with polytherapy.

\begin{tabular}{|c|c|c|}
\hline Parameter & Amlodipine monotherapy $(\mathrm{N}=13)$ & Polytherapy $(\mathrm{N}=27)$ \\
\hline Carotid-femoral PWV $(\mathrm{cm} / \mathrm{s})$ & $989.83 \pm 460.33$ & $1030.8 \pm 402.56$ \\
\hline Left brachial PWV $(\mathrm{cm} / \mathrm{s})$ & $864.59 \pm 1796.0$ & $1165.4 \pm 1397.1$ \\
\hline Right brachial PWV (cm/s) & $1498.5 \pm 693.97$ & $15137 \pm 523.41$ \\
\hline Right brachial ASI (mmHg) & $22.715 \pm 14.696$ & $23.626 \pm 11.850$ \\
\hline Left brachial ASI (mmHg) & $25.731 \pm 7.041$ & $27.185 \pm 9.638$ \\
\hline Right ankle ASI (mmHg) & $36.000 \pm 9.410$ & $36.470 \pm 10.990$ \\
\hline Left ankle ASI (mmHg) & $40.054 \pm 7.241$ & $38.948 \pm 9.188$ \\
\hline Pulse pressure (mmHg) & $57.65 \pm 13.17$ & $59.64 \pm 16.81$ \\
\hline
\end{tabular}

PWV: pulse wave velocity; ASI: arterial stiffness index; data are presented as mean \pm SD; polytherapy group, included patients taking ACE inhibitors, beta blockers and statins; Mann Whitney test was used for comparison. 
and pulse pressure were significantly higher among the hypertensives as compared to normotensives. Body mass index (BMI) may also contribute to the occurrence of arterial stiffness; however BMI was not significantly different among the groups in the present study [16]. Smoking and caffeine can adversely affect vascular stiffness both acutely and chronically [17]. Hence care was taken to ensure that subjects were free of smoking and caffeine containing agents at least $2 \mathrm{hrs}$ before the recording of the vascular parameters. Further smoking status was not statistically different among the three groups.

Increased vascular stiffness among chronic and newly diagnosed hypertensives emphasizes the importance of early detection of blood pressure and vascular stiffness in addition to adequate control of blood pressure. Currently there are no guidelines for the use of specific drugs to target and improve arterial stiffness. However, there is evidence that adequate control of blood pressure itself is a first step to treat the vascular stiffness. Moreover, ACE-inhibitors, angiotensin II receptor blockers, aldosterone receptor antagonists and calcium channel blockers have shown to have favorable effects compared to beta blockers [18]. Hence larger studies are needed to determine the normal reference values of the vascular parameters of arterial stiffness in Indian population, which would help to establish guidelines on use of drugs targeting the arterial stiffness and utility of these parameters in long term prognosis of hypertension.

\section{Limitation of the Study}

The major limitation of our study is the small sample size. The effect of drugs on vascular stiffness in chronic hypertensives was studied in a small sample of forty patients. These results need to be tested further in larger population studies. Background therapy was not uniform in the chronic as well as newer hypertensive patients.

As our study is cross sectional it only provides the data of vascular stiffness in known and newly diagnosed hypertensives as compared to controls. Further, among hypertensives prospective blood samples were not collected for vascular inflammatory markers. Lipid profiles were not available for all subjects.

\section{Conclusion}

PWV, ASI and pulse pressure are significantly higher in chronic and newly diagnosed non-diabetic hypertensives as compared to controls irrespective of their treatment status.

\section{Acknowledgements}

We thank Indian Council of Medical Research, for the grant of Short Term Studentship for the study (Reference ID 2012-02485).

\section{References}

[1] WHO (2012) World Health Report 2002 Press Kit. http://www.who.int/whr/2002/media_centre/en/index.html

[2] WHO (2012) World Health Statistics 2008. http://www.who.int/gho/publications/world_health_statistics/EN_WHS08_Full.pdf

[3] Kearney, P.M., Whelton, M., Reynolds, K., Muntner, P., Whelton, P.K. and He, J. (2005) Global Burden of Hypertension: Analysis of Worldwide Data. Lancet, 365, 217-223. http://dx.doi.org/10.1016/S0140-6736(05)17741-1

[4] Howell, S.J., Sear, J.W. and Foëx, P. (2004) Hypertension, Hypertensive Heart Disease and Perioperative Cardiac Risk. British Journal of Anaesthesia, 92, 570-583. http://dx.doi.org/10.1093/bja/aeh091

[5] Laurent, S., Boutouyrie, P., Asmar, R., Gautier, I., Laloux, B., Guize, L., et al. (2001) Aortic Stiffness Is an Independent Predictor of All-Cause and Cardiovascular Mortality in Hypertensive Patient. Hypertension, 37, 1236-1241. http://dx.doi.org/10.1161/01.HYP.37.5.1236

[6] Laurent, S., Cockcroft, J., Van Bortel, L., Boutouyrie, P., Giannattasio, C., Hayoz, D., et al. (2006) Expert Consensus Document on Arterial Stiffness: Methodological Issues and Clinical Applications. European Network for Non-Invasive Investigation of Large Arteries. European Heart Journal, 27, 2588-2605. http://dx.doi.org/10.1093/eurheartj/ehl254

[7] Palatini, P., Casiglia, E., Gąsowski, J., Głuszek, J., Jankowski, P., Narkiewicz, K., et al. (2011) Arterial Stiffness, Central Hemodynamics, and Cardiovascular Risk in Hypertension. Vascular Health and Risk Management, 7, 725-739. http://dx.doi.org/10.2147/VHRM.S25270

[8] Dolan, E., Thijs, L., Li, Y., Atkins, N., McCormack, P., McClory, S., et al. (2006) Ambulatory Arterial Stiffness Index as a Predictor of Cardiovascular Mortality in the Outcome Study. Hypertension, 47, 365-370. 
http://dx.doi.org/10.1161/01.HYP.0000200699.74641.c5

[9] Blacher, J., Asmar, R., Djane, S., London, G.M. and Safar, M.E. (1999) Aortic Pulse Wave Velocity as a Marker of Cardiovascular Risk in Hypertensive Patients. Hypertension, 33, 1111-1117. http://dx.doi.org/10.1161/01.HYP.33.5.1111

[10] Willum-Hansen, T., Staessen, J.A., Torp-Pedersen, C., Rasmussen, S., Thijs, L., Ibsen, H., et al. (2006) Prognostic Value of Aortic Pulse Wave Velocity as Index of Arterial Stiffness in the General Population. Circulation, 113, 664670. http://dx.doi.org/10.1161/CIRCULATIONAHA.105.579342

[11] Benetos, M.S., Rudnichi, A., Smulyan, H., Richard, J.L., Ducimetieère, P., et al. (1997) Pulse Pressure. A Predictor of Long-Term Cardiovascular Mortality in a French Male Population. Hypertension, 30, 1410-1415. http://dx.doi.org/10.1161/01.HYP.30.6.1410

[12] Naidu, M.U., Reddy, B.M., Yashmaina, S., Patnaik, A.N. and Rani, P.U. (2005) Validity and Reproducibility of Arterial Pulse Wave Velocity Measurement Using New Device with Oscillometric Technique: A Pilot Study. BioMedical Engineering OnLine, 4, 49. http://dx.doi.org/10.1186/1475-925X-4-49

[13] Arcaro, G., Laurent, S., Jondeau, G., Hoeks, A.P. and Safar, M.E. (1991) Stiffness of the Common Carotid Artery in Treated Hypertensive Patients. Journal of Hypertension, 9, 947-954. http://dx.doi.org/10.1097/00004872-199110000-00008

[14] Asmar, R. (2001) Effect of Antihypertensive Agents on Arterial Stiffness as Evaluated by Pulse Wave Velocity: Clinical Implications. American Journal of Cardiovascular Drugs, 1, 387-397. http://dx.doi.org/10.2165/00129784-200101050-00008

[15] Benetos, B.W., Izzo, J., Mitchell, G., Resnick, L., Asmar, R., et al. (2002) Influence of Age, Risk Factors, and Cardiovascular and Renal Disease on Arterial Stiffness: Clinical Applications. American Journal of Hypertension, 15, 1101 1108. http://dx.doi.org/10.1016/S0895-7061(02)03029-7

[16] Nordstrand, N., Gjevestad, E., Dinh, K.N., Hofsø, D., Røislien, J. and Saltvedt, E. (2011) The Relationship between Various Measures of Obesity and Arterial Stiffness in Morbidly Obese Patients. BMC Cardiovascular Disorders, $11,7$. http://dx.doi.org/10.1186/1471-2261-11-7

[17] Vlachopoulos, C., Kosmopoulou, F., Panagiotakos, D., Ioakeimidis, N., Alexopoulos, N., Pitsavos, C., et al. (2004) Smoking and Caffeine Have a Synergistic Detrimental Effect on Aortic Stiffness and Wave Reflections. Journal of the American College of Cardiology, 44, 1911-1917. http://dx.doi.org/10.1016/j.jacc.2004.07.049

[18] Duprez, D.A. (2010) Is Vascular Stiffness a Target for Therapy? Cardiovascular Drugs and Therapy, 24, $305-310$. http://dx.doi.org/10.1007/s10557-010-6250-Z 